\title{
Effect of Hydrogen Bonding on Linear and Nonlinear Rheology of Entangled Polymer Melts
}

Shabbir, Aamir; Goldansaz, Hadi; Hassager, Ole; van Ruymbeke, Evelyne; Alvarez, Nicolas J.

Published in:

Macromolecules

Link to article, DOI:

10.1021/acs.macromol.5b00757

Publication date:

2015

Document Version

Peer reviewed version

Link back to DTU Orbit

Citation (APA):

Shabbir, A., Goldansaz, H., Hassager, O., van Ruymbeke, E., \& Alvarez, N. J. (2015). Effect of Hydrogen Bonding on Linear and Nonlinear Rheology of Entangled Polymer Melts. Macromolecules, 48(16), 5988-5996. https://doi.org/10.1021/acs.macromol.5b00757

\section{General rights}

Copyright and moral rights for the publications made accessible in the public portal are retained by the authors and/or other copyright owners and it is a condition of accessing publications that users recognise and abide by the legal requirements associated with these rights.

- Users may download and print one copy of any publication from the public portal for the purpose of private study or research.

- You may not further distribute the material or use it for any profit-making activity or commercial gain

- You may freely distribute the URL identifying the publication in the public portal 


\author{
Aamir Shabbir, ${ }^{\dagger}$ Hadi Goldansaz, ${ }^{\ddagger}$ Ole Hassager, $₫$ Evelyne Van Ruymbeke, ${ }^{\ddagger}$ and \\ Nicolas J. Alvarez ${ }^{*}$, \\ Department of Chemical and Biochemical Engineering - Lyngby Denmark, Bio and Soft \\ Matter Division (BSMA), Institut de la Matire Condense et des Nanosciences (IMCN), \\ Louvain-la-Neuve, Belgium, Department of Chemical and Biochemical Engineering, Lyngby, \\ Denmark, and Department of Chemical and Biological Engineering, Philadelphia, USA \\ E-mail: alvarez@drexel.edu
}

\begin{abstract}
Supramolecular polymers are used in many applications such as adhesives, coatings, cosmetics, and printing. Characterizing the dynamics of such polymers is essential for tailoring user defined properties in products and applications. We present both linear and nonlinear rheological results for a model system of pure poly(n-butyl acrylate), PnBA, homopolymer and four PnBA-poly(acrylic acid), PnBA-PAA, copolymers with different number of AA side groups. The copolymers are synthesized via hydrolysis of the pure PnBA homopolymer. Therefore, all polymers studied have the same backbone length. The number of AA side groups (hydrogen bonding groups) after hydrolysis is determined from NMR measurements. We show that using the theoretical dependency
\end{abstract}

\footnotetext{
*To whom correspondence should be addressed

†Technical University of Denmark

†Université catholique de Louvain

I Technical University of Denmark

$\S$ Drexel University
} 
of modulus and reptation time on the packing length, we can account for the changes in linear viscoelasticity due to transformation of $\mathrm{nBA}$ side groups to AA along the backbone. Assuming superposition holds and subtracting out the linear chain rheology from LVE, the hydrogen bonding contribution to LVE is exposed. Hydrogen bonding affects linear viscoelasticity at frequencies below the inverse reptation time. More specifically, the presence of hydrogen bonds causes $G^{\prime}$ and $G^{\prime \prime}$ as a function of frequency to shift to a power law scaling of 0.5. Furthermore, the magnitude of $G^{\prime}$ and $G^{\prime \prime}$ scales linearly with the number of hydrogen bonding groups. The nonlinear extensional rheology shows extreme strain hardening. The magnitude of extensional stress has a strongly nonlinear dependence on the number of hydrogen bonding groups. These results are aimed at uncovering the molecular influence of hydrogen bonding on linear and nonlinear rheology to aid future molecular synthesis and model development.

\section{Introduction}

Supramolecular polymers represent an emerging class of polymers that offer superior material properties compared to their non-associating counterparts. Supramolecular polymers are a broad category of materials that include all polymers whose monomeric units can associate via secondary interactions. These secondary interactions can be due to hydrogen bonding ${ }^{1-7}$ , metal-ligand bonding, ${ }^{8,9}$ and/or ionic bonding. ${ }^{10-13}$ Hydrogen bonding is the most common non-covalent reversible interaction employed to create supramolecular polymeric assemblies ${ }^{3}$. Although hydrogen bonds form one of the weakest noncovalent interactions, their ability to form highly directional and versatile associations make them very useful. ${ }^{14}$ For example, cooperative hydrogen bonding is responsible for the material properties of nylons.

The association strength of a single hydrogen bond in a polymer melt (no solvent effects) depends on the nature of donor and acceptor. The association strength of multiple hydrogen bonding units depends on the same, but also the number and arrangement of the hydrogen bonds. ${ }^{15,16}$ One particularly useful trait of hydrogen bonds is that the association 
strength and dynamics can be significantly altered via external stimuli, e.g. temperature. At high temperatures, the association strength of a hydrogen bond is significantly reduced and thus the presence of hydrogen bonds has little to no effect on material properties. At low temperatures, the association strength of the hydrogen bond is large and the lifetime is such that the bonds act like weak crosslinks between chains. ${ }^{1}$ This ability to thermally tune association strengths allows for easy processing of such materials for various applications. ${ }^{17}$

Although a large body of work on H-bonding supramolecular polymer synthesis exists in the literature, very few studies have examined the mechanical properties of these polymers in the melt state. ${ }^{1,5,15,18}$ Lewis and coworkers in 2014 reported the influence of density, molecular structure, and association strength considering four different hydrogen bonding groups on linear viscoelasticity. The authors determine that the presence of hydrogen bonds (i) elevates the material's glass transition termperature, (ii) copolymers with weakly associating hydrogen bonds (acrylic acid (AA), carboxyethylacrylate (CEA), acrylamidopyridine (AP)) show no evidence of network formation, and finally (iii) conclude that side-group dimerization alone is not sufficient to form a mechanically relevant network. ${ }^{1}$ Almost two decades prior, Stadler and coworkers examined the linear viscoelastic properties of 1,2,4-triazoline-3,5-dione (TAD) modified polybutadiene following a procedure by Leong and Butler. ${ }^{19,20}$ From this work, the LVE properties of the modified polybutadiene shows three characteristics as a function of the number of hydrogen bonding groups (i) increase in plateau modulus, (ii) decrease in the low frequency crossover $\omega_{L}$, and (iii) a transition in power law scaling of $G^{\prime}$ and $G^{\prime \prime}$ from 2 and 1 , respectively to $\approx 0.5$ in the LVE terminal regime. It is not clear from this data since the addition of TAD highly modifies the backbone chemistry by eliminating double bonds, whether all three effects are due to either hydrogen bonding, change in chemistry, or a combination of both. We agree with the comment by Lewis et al. that comparative studies involving a single backbone are necessary to elucidate a clear structure-property relationship in hydrogen bonding polymers. ${ }^{1}$

In this work, we use a model entangled monodisperse linear PnBA polymer, which is 
selectively hydrolyzed to convert nBA side groups to AA side groups, in order to isolate the effect of hydrogen bonding on linear viscoelasticity of entangled polymer melts. More specifically, we examine four PnBA-PAA copolymers with increasing number of acrylic acid groups: pure PnBA, 6\% AA, 13\% AA, and 38\% AA. The percentage of AA is determined post-hydrolysis using NMR spectroscopy. Since all polymers are synthesized from the same starting PnBA and hydrolysis does not change the chemistry of the backbone, the four polymers have the same backbone chemistry and length. Using a simple model that takes into account changes in packing length, we convincingly show that the contribution of hydrogen bonding to linear viscelasticity is restricted to the viscous terminal regime. In other words, hydrogen bonding only hinders reptation of the entangled chains and at a critical number of AA side groups forms a weak gel at low frequencies. Using this understanding, we probe the nonlinear mechanical properties of the four systems using a filament stretching rheometer. We show that the hydrogen bonds give rise to extreme strain hardening behavior when the strain rates are significantly below the inverse reptation time. Both the linear and nonlinear results are put into the context of the state of the art.

\section{Experimental Details}

\section{Materials}

Poly(n-butyl acrylate) (PnBA) was used as received from Polymer Source Inc. (Product No. P1111-nBUA lot 18124). The PnBA is reported by supplier to have a $\mathrm{Mn}=193 \mathrm{k}, \mathrm{Mw}=210 \mathrm{k}$, and PDI=1.085. The Molecular weight was remeasured in-house via a size exclusion chromatography setup consisting of a SIL-10AD injector (Shimadzu), a triple Viscotek detector and two PLgel Mixed C and Mixed D columns. Stabilized tetrahydrofuran (THF) was used as the eluent and the flow rates have been adjusted according to Irganox signals. The columns were calibrated using PS standards. The PnBA was measured to have Mn=121k, $\mathrm{Mw}=166 \mathrm{k}$, and a PDI=1.375. Using the entanglement molecular weight specified by Jullian 
and coworkers, $M_{e}=32 \mathrm{~kg} / \mathrm{mol},{ }^{21}$ then the number of entanglements for the pure PnBA is $Z \approx 5$. Three partially hydrolyzed samples of PnBA were produced by dissolving as received $1 \mathrm{~g}$ PnBA in $30 \mathrm{~mL}$ distilled THF. $200 \mathrm{mg}$ anhydrous potassium hydroxide (KOH) was added to the PnBA solution. The hydrolysis reaction proceeded under reflux conditions at $70^{\circ} \mathrm{C}$ and argon atmosphere. After a desired time, the reaction mixtures were cooled to room temperature and neutralized with excess amount of $\mathrm{HCl}$. The amount of hydrolyzed PnBA groups depends on the duration of hydrolyzation. After hydrolyzation, the samples were dried using a rotary evaporator to ensure complete removal of n-butanol and THF. To remove potassium chloride salt, the dried polymer samples were dissolved in $30 \mathrm{ml}$ ethyl acetate and $30 \mathrm{ml}$ of ultra-pure water. The mixture was stirred for $10 \mathrm{~min}$ and the ethyl acetate was removed by rotary evaporator, which forces the polymer to precipitate. This washing step was repeated 4-5 times to ensure complete removal of the inorganic salt. Finally, the polymer samples were dried under vacuum at $50^{\circ} \mathrm{C}$ for 15 days. Extent of reaction, i.e. the number of nBA side groups hydrolyzed to AA, was quantified using ${ }^{1} H \mathrm{NMR}$ spectroscopy.

The extent of hydrolysis was determined by ${ }^{1} \mathrm{H}$ NMR in solution by analyzing $10 \mathrm{mg}$ polymer in $0.6 \mathrm{ml}$ of $\mathrm{CDCl} 3$ using $>200$ scans separated by $>25$ s recycling time. A Bruker AVANCE $500 \mathrm{MHz}$ spectrometer was used for recording ${ }^{1} \mathrm{H}-\mathrm{NMR}$ spectra. The average fraction of hydrolyzed monomers was determined by using the ratio of triplet methyl protons of the butyl side chain $(<0.9 \mathrm{ppm})$ and methylene protons of the butyl side chains next to the ester $(\approx 4.3 \mathrm{ppm})$ to the rest of the protons of the backbone and side chain $(1<$ chemical shift $<2.4 \mathrm{ppm})$.

In the presence of metallic salts, such hydrogen bonding systems behave like ionomers, in which acid groups are adsorbed to the ionic aggregates. In carboxylic acid containing ionomers, the association strength and consequently both linear and non-linear rheology strongly depend on the nature of the cation as well as the counter ion. Hence it is imperative for the purpose of this study to purify the sample from metallic contaminations, before any measurements. Purification was performed by successive washing of the sam- 
ples. Energy dispersive X-ray spectrometry analysis (EDX) was used in order to quantify the residual metallic contamination after synthesis and purification of the copolymers. EDX measurements were carried out using a JEOL FEG SEM 7600F scanning electron microscope equipped with an EDX system (Jeol JSM2300 with a resolution <129 eV) operating at 15 $\mathrm{keV}$ with a working distance of $8 \mathrm{~mm}$. The acquisition time for the chemical spectra lasted $300 \mathrm{~s}$ with a probe current of $1 \mathrm{nA}$. Specimens for EDX were mounted on stubs and analyzed without metallization. All samples showed less than $0.6 \%$ metallic content after washing. Most samples showed less athan $0.1 \%$ metallic content after washing.

The samples and their characteristics are shown in Table 1 . Given that the $Z=5$, an examination of Table 1 shows that even for $\operatorname{Ref}(\mathrm{PnBA})$ there are several AA groups per entanglement section.

Table 1: Percentage and number of AA side groups on backbone

\begin{tabular}{lcc}
\hline \hline Sample Name & \%sticky groups/chain & Number of AA side groups/chain \\
\hline Ref (PnBA) & $3 \pm 2 \%$ & 49 \\
AA6 & $6 \pm 2 \%$ & 90 \\
AA13 & $13 \pm 2 \%$ & 207 \\
AA38 & $38 \pm 2 \%$ & 615 \\
\hline \hline
\end{tabular}

\section{Rheology}

\section{Linear Viscoelasticity}

Linear visoelastic properties of the PnBA-AA copolymers were investigated by small amplitude oscillatory shear (SAOS) rheology using a MCR 301 (Anton Paar, Germany) rheometer. Temperature was controlled using a convection oven operating under nitrogen. Three different $(8,15$ or $20 \mathrm{~mm}$-diameter) parallel plate geometries were used depending on the viscosity level of the system at the given temperature. Furthermore, the different geometries were used to ensure proper accounting for tool compliance. No slippage problem was observed due to good adhesion of the polymers to the surface of the steel geometries. For samples pure

\section{ACS Paragon Plus Environment}


PnBA, AA6, the linear viscoelastic data were measured between $-25^{\circ} \mathrm{C}$ and $45^{\circ} \mathrm{C}$ and time temperature superposition was used to create master curves. For samples AA13 and AA38, the linear viscoelastic data were measured between $-15^{\circ} \mathrm{C}$ and $30^{\circ} \mathrm{C}$ and time temperature superposition was used to create master curves. Outside of this range (more specifically above $30^{\circ} \mathrm{C}$ ) samples with acrylic acid content above $6 \%$ show thixotropic behavior, do not follow TTS, and therefore are not included in the generation of a master curve. The thixotropic behavior of these samples is a discussion for a future article.

\section{Extensional viscosity measurements}

The extensional stress growth coefficient as a function of time was measured using a filament stretching rheometer (DTU-FSR). Cylindrical stainless steel sample plates with a diameter of $5.4 \mathrm{~mm}$ were used for all measurements. The mass of each sample varied from $0.04 \mathrm{~g}$ to $0.06 \mathrm{~g}$. The aspect ratio, $\Lambda_{0}=L_{0} / R_{0}$, varied from 0.36 to 0.75 . Measurements were performed at a constant Hencky strain rate imposed at the mid-filament diameter using an online control scheme. ${ }^{22}$ All experiments were performed at $21.5^{\circ} \mathrm{C}$. The imposed strain rates were varied from $0.0006 s^{-1}$ to $1 s^{-1}$. All samples except AA38, were relatively fluid like and were formed into an approximate cylindrical test specimen manually using a steel spatula. After considerable time, the sample relaxed to form an axisymmetric cylindrical shape due to surface tension. It is very important that sufficient time is allowed for the sample to completely relax before performing any measurement. This was ensured by monitoring the force on the sample and waiting for the force to be sufficiently close to zero, i.e. zero within the noise $( \pm 0.05 \mathrm{~g})$ of the force transducer. Since sample AA38 has extremely long relaxation times (see LVE data in Results), it was annealed at $80^{\circ} \mathrm{C}$ under a nitrogen rich environment for approximately half an hour. Nitrogen was used during annealing to prevent sample oxidation. Prior to performing elongational measurements, sample AA38 was pre-stretched at $80^{\circ} \mathrm{C}$ to a diameter between $2.48 \mathrm{~mm}$ and $4.4 \mathrm{~mm}$ depending on the applied strain rate. At which point, the temperature was reduced to the desired temperature and the stress was 
allowed to relax for up to 2 hours depending on the sample. Such practice ensures that the maximum force during the experiment was low enough to avoid sample detachment from the plates.

\section{Results}

The linear viscoelasticity (LVE) measured using small amplitude oscillatory shear is presented in Fig. 1 for all four samples. Fig. 1a shows the response for the PnBA as received from the supplier. At high frequency, $G^{\prime}$ and $G^{\prime \prime}$ show the classical frequency dependence expected for a monodisperse entangled linear polymer melt. There is a power law dependence of $G^{\prime \prime}$ in the glassy regime near the high-frequency crossover, $\omega_{H}$, a discernible plateau modulus in $G^{\prime}$, and an inflection in $G^{\prime \prime}$ just before the low frequency cross-over, $\omega_{L}$. However, at frequencies below $\omega_{L}$, known as the terminal regime, $G^{\prime}$ and $G^{\prime \prime}$ deviate from the expected power law dependence of two and one, respectively. This deviation is surprising to the authors, since no data for a monodisperse entangled linear polymer melt is known to show such a deviation in the terminal regime. Two explanations for the observed discrepancy are, (i) the relatively large PDI, measured via SEC measurements, is too broad to expect a monodisperse rheological response or (ii) the discrepancy is due to hydrogen bonding via the small amount of AA/chain, 3\%, present in the sample measured via NMR, see Table 1. We will show that reasoning (ii) is the most likely explanation.

Fig. 1b shows LVE data for sample AA6, which is composed of $6 \%$ AA along the backbone. Even though the amount of AA is doubled, Fig. 1b shows very similar LVE behavior to the PnBA sample in Fig. 1a. Qualitatively, the only difference between the two data sets appears to be a slight shift in the frequency dependence of $G^{\prime}$ and $G^{\prime \prime}$ at frequencies below, $\omega_{L}$. Fig. 1c, shows LVE data for sample AA13, which is composed of $13 \%$ AA along the backbone. Again Fig. 1c shows similar LVE behavior at high frequency, but a much more pronounced change in low frequency dynamics. The greatest change in LVE behavior 


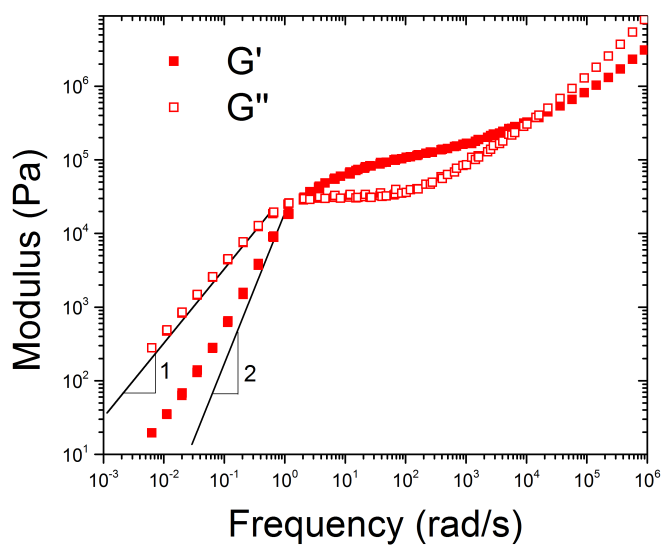

(a) PnBA

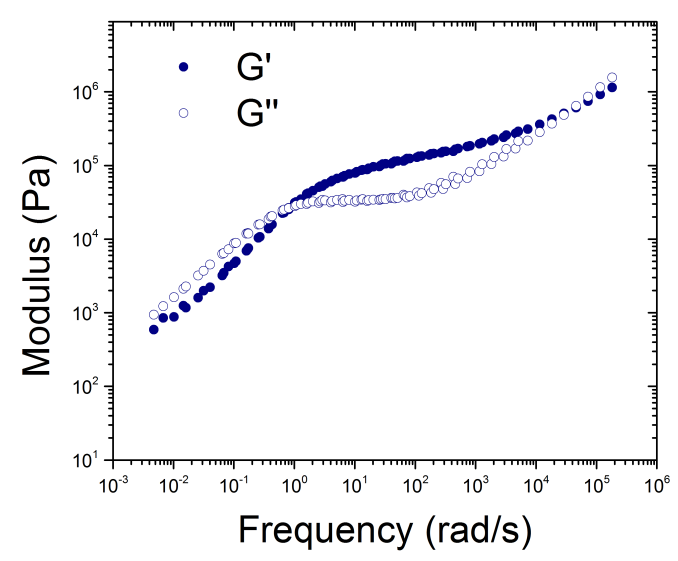

(c) AA13, $13 \% \mathrm{AA}$

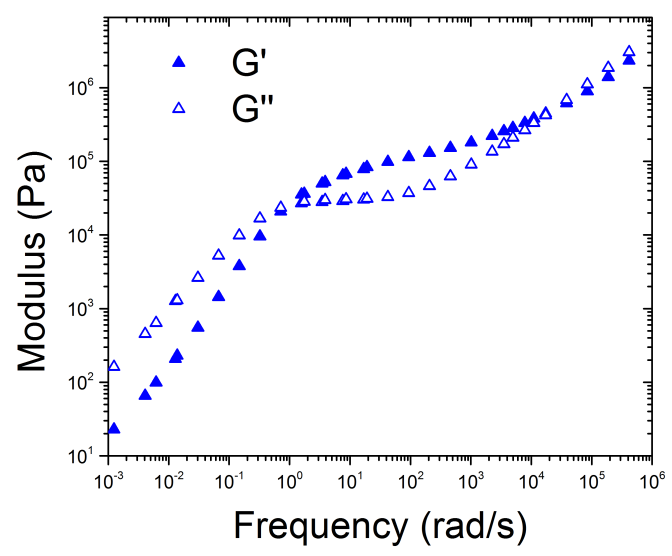

(b) AA6, 6\% AA

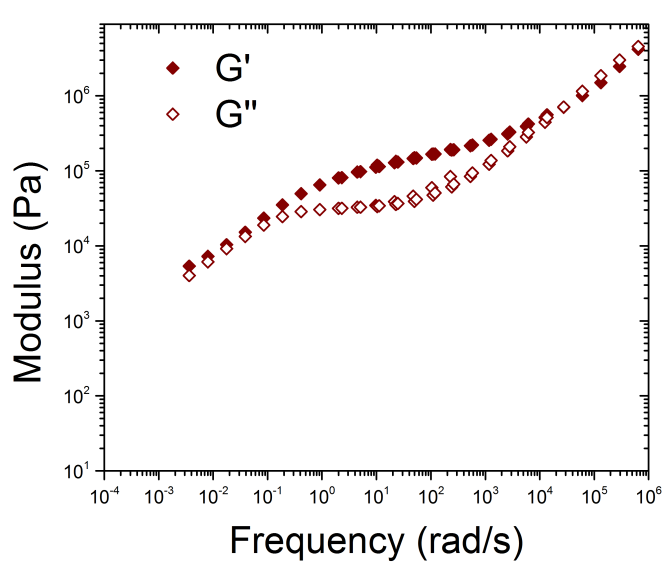

(d) AA38, 38\% AA

Figure 1: shows the linear viscoelastic response of all four samples measured using small amplitude oscillatory shear. The lines in (a) represent the expected power law dependence of $G^{\prime}$ and $G^{\prime \prime}$ for a monodisperse linear polymer melt in the low frequency, terminal regime.

is shown in Fig. 1d, for sample AA38, wherby $G^{\prime}$ and $G^{\prime \prime}$ lie parallel and on top of each other for low frequencies. This is indicative of a weak network, i.e. gel, or Rouse dynamics. It would appear from Figs. 1a-1d that the existence of AA side groups strongly alter the behavior of $G^{\prime}$ and $G^{\prime \prime}$ at frequencies lower than $\omega_{L}$.

Fig. 2 shows a closer examination of (a) the elastic modulus and (b) the viscous modulus. What is now evident from Fig. 2a is that there is a slight shift in the plateau modulus with increasing AA content. Furthermore, by comparing Fig. 2a and $2 \mathrm{~b}$ there is a shift in the low frequency crossover. Therefore, hydrolyzing the nBA side group to AA along the backbone 


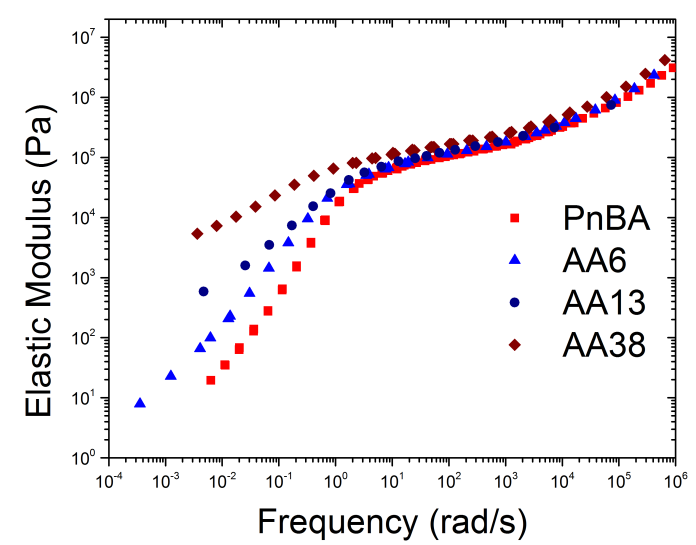

(a) Elastic Modulus

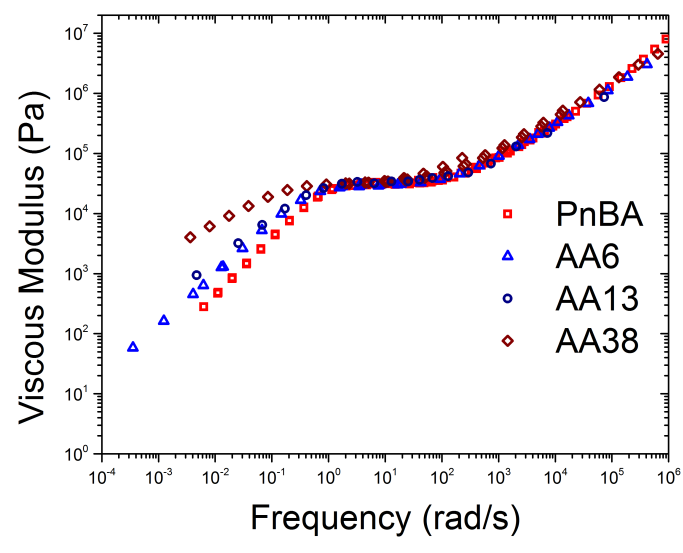

(b) Viscous Modulus

Figure 2: Shows an overlay of all data presented in Fig 1 split into (a) $G^{\prime}$, elastic modulus, and (ii) $G^{\prime \prime}$, viscous modulus.

has the following three effects on LVE, (i) increases the plateau modulus, (ii) decreases the low frequency crossover, $\omega_{L}$, and (iii) changes the power law dependence and increases the overall magnitude of $G^{\prime}$ and $G^{\prime \prime}$ at frequencies lower than $\omega_{L}$. These three dependencies will be examined in greater detail in the discussion section.

We now examine the extensional rheology of the four samples in the context of the LVE data presented above. Fig. 3 shows the extensional rheology of all four samples for various strain rates. The strain rates are always increasing from right to left, i.e. the farthest curve to the right in Fig. 3a corresponds to the smallest strain rate $0.3 \mathrm{~s}^{-1}$ and the farthest to the left corresponds to the highest strain rate $3 \mathrm{~s}^{-1}$. The solid line in all figures represent the corresponding LVE envelope determined from a multi-mode Maxwell fit to the LVE data shown in Fig. 1, see supplementary for best fit parameters. It is evident from the solid lines in Figs. 3b-3d that the shear viscosity is increasing with increasing AA content. Note that a terminal regime was not observed in the LVE data and therefore a zero shear rate viscosity cannot be reported. Thus, any flattening observed in the LVE envelope (solid lines) is due to a finite number of modes used to fit the linear viscoelastic data and not the asymptotic approach to a zero shear rate viscosity. The dashed lines in Fig. 3b-3d represents the LVE envelope for PnBA, i.e. the solid line shown in Fig. 3a is replicated for reference in all three 


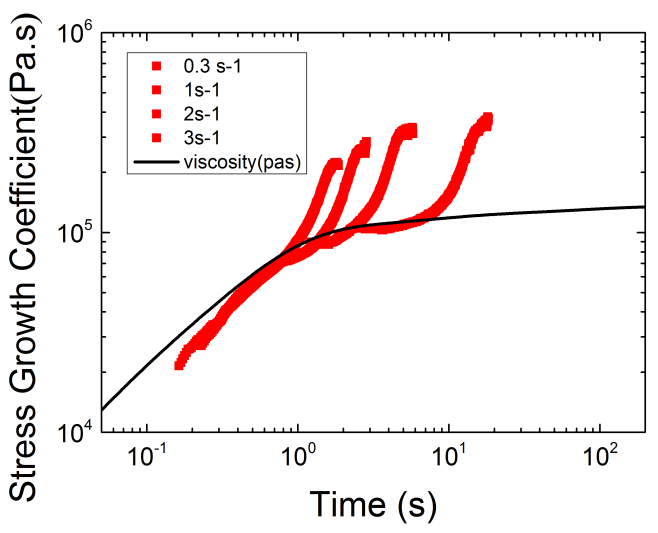

(a) PnBA

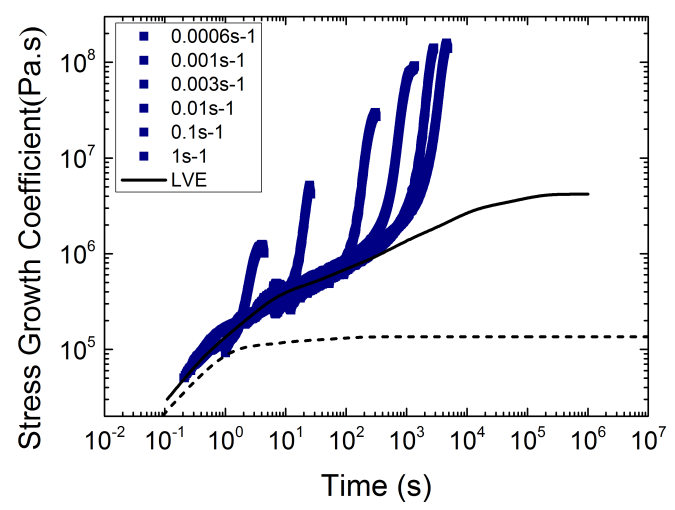

(c) AA13, $13 \%$ AA

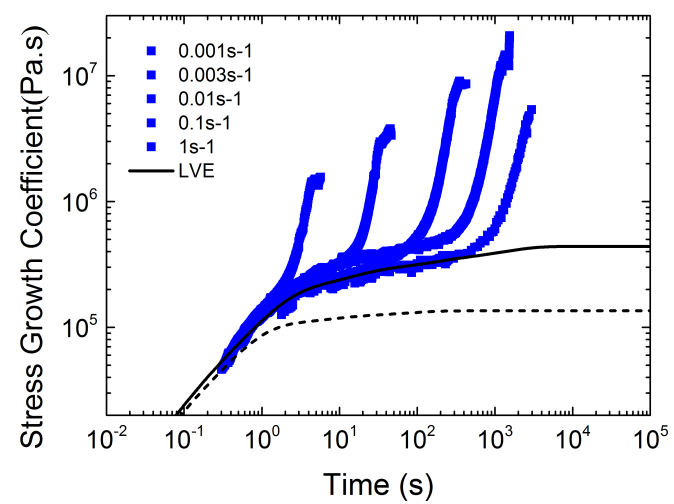

(b) AA6, 6\% AA

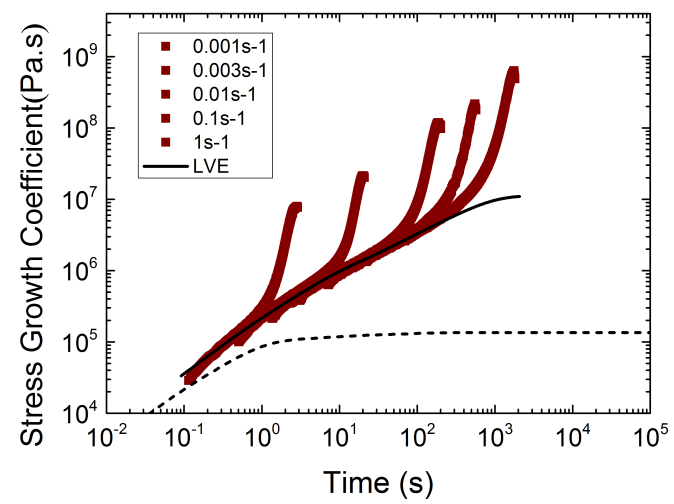

(d) AA38, 38\% AA

Figure 3: shows the stress growth coefficient measured using the filament stretching rheometer. The solid lines represent the best fit of the multi-mode Maxwell model to the LVE data shown in Fig. 1. The dashed lines in (b)-(d) represent the multi-mode Maxwell model for the PnBA sample (solid line in (a)) for comparison.

figures. Typically in extensional rheology of monodisperse entangled linear polymer melts, the measured stress growth coefficient follows the LVE envelope for strain rates much less than the inverse Rouse time, $1 / \tau_{R}$. For strain rates greater than $1 /\left(0.5 \tau_{R}\right)$, the stress growth coefficient deviates upwards from the LVE envelope. This upwards deviation is referred to as strain hardening. $\tau_{R}=0.038 \mathrm{~s}$ for the PnBA studied here, which is calculated using BSW parameters, the BSW model and parameter estimation are explained in more detail in the discussion section. Thus, for PnBA, strain hardening is not expected for strain rates $\dot{\epsilon}<13$ $\mathrm{s}^{-1}$. It is convenient to discuss the relative magnitude of $\dot{\epsilon}$ to $\tau_{R}$ in terms of the Weissenberg 
number, $W i=\dot{\epsilon} \tau_{R}$ i.e. when $W i<0.5$ no strain hardening is expected and when $W i \geq 0.5$ strain hardening is expected. It is evident from Fig. 3a that even for the lowest $W i=0.01$, strain rate $0.3 \mathrm{~s}^{-1}$, strain hardening is observed. The strain hardening plateaus at a value approximately a factor of three higher than the zero shear rate viscosity. We hypothesize that this deviation is most likely due to the small presence of $\mathrm{AA}, 3 \%$, in the PnBA as received. Note that if this is indeed the case, one should be careful to use PnBA as a model linear polymer system assuming no association groups. ${ }^{23}$

Fig 3b shows the extensional rheology of sample AA6 for five increasing strain rates from 0.001-1 s ${ }^{-1}$. At small times, all curves follow the LVE envelop. At larger times there is a sharp strain hardening behavior observed for all measured strain rates. The strain hardening achieves a magnitude an order higher than the LVE envelope and two orders of magnitude higher than the zero shear rate viscosity of PnBA denoted by the dotted line. The same strain hardening behavior is observed for samples AA13 and AA38, see Fig. 3c-3d. The main difference is that a doubling of the number of AA side groups per chain causes an order of magnitude increase in the strain hardening behavior. Sample AA6, AA13 and AA38 achieve stress growth coefficients two, three and four orders of magnitude above the zero shear rate viscosity of PnBA, respectively.

It is important to note that the greatest strain hardening behavior is observed for strain rates much lower than the strain rates applied to PnBA in Fig. 3a. Assuming the same Rouse time for all samples, $W i \leq 4 \times 10^{-5}$ for the lowest strain rates in Fig 3b-3d. By calculating $W i$ consider the Rouse time of pure PnBA, we are putting the results in perspective of what would be the expected nonlinear behavior of a linear chain. For such extremely low Wi, the stress growth coefficient is expected to follow the LVE envelope for all relevant strains if this were a non-associating linear chain. The significant deviation from LVE of pure PnBA demonstrates the influence of hydrogen bonding on the nonlinear response of an entangled linear polymer melt at low strain rates and thus effectively increasing the characteristic Rouse time of the material. In other words, addition of hydrogen bonding side groups help 
to prevent creeping of the material.

\section{Discussion}

It was noted above that hydrolyzation of nBA to AA affected the LVE behavior by (i) increasing the plateau modulus, $G_{N 0}$, (ii) decreasing the low frequency crossover, $\omega_{L}$, and (iii) increasing $G^{\prime}$ and $G^{\prime \prime}$ and altering their power law dependence at frequencies lower than $\omega_{L}$. Recall that these are the same three observations drawn from the work of Stadler and Freitas regarding modified polybutadienes. ${ }^{19}$ At least for these two systems, these observations seem to be general and independent of chemistry. We are interested in discerning which of these three effects can be attributed to introduction of hydrogen bonding via exposure of the acrylic group and which are the result of the reduction in effective chain diameter. To analyze this question, we make the assumption that hydrogen bonding and changes in effective diameter affect linear viscoelasticity independently and thus are additive effects. Since all hydrolyzed samples are derived from the same PnBA molecule the changes in chemistry can be accounted for by taking into account changes in packing length of the polymer molecule. The packing length of a polymer chain is given by the equation,

$$
P=\frac{\nu_{0}}{b^{2}}
$$

where $\nu_{0}$ is the volume of the polymer repeat unit and $b$ is the Kuhn length. Since $\nu_{0}$ depends on the molecular weight of the repeat unit, then it is expected that the packing length will depend on the percentage of nBA hydrolyzed to AA. We assume that since the backbone chemistry is left unchanged via hydrolysis the kuhn length is not a function of the number of AA side groups (see argument in preceding paragraph). The molecular weight of a nBA repeat unit is $M_{n B A}=128 \mathrm{~g} / \mathrm{mol}$. After hydrolysis, the $\mathrm{nBA}$ is converted to AA with a a molecular weight of $M_{A A}=72 \mathrm{~g} / \mathrm{mol}$. The $\mathrm{Mw}$ of the chain after hydrolysis is given by 
$M w_{H}=\alpha M w$, where

$$
\alpha=(1-0.44 f)
$$

where $f$ is the mol fraction of AA side groups and $\alpha$ represents the transition in $\mathrm{Mw}$ from pure nBA, $\alpha=1$, to pure AA, $\alpha=0.56$. Note that in what follows the superscript " 0 " denotes the property with respect to pure PnBA. We therefore define an average packing length per chain for a given fraction of AA,

$$
\bar{P}=\frac{\alpha \nu_{0}}{b^{2}}
$$

Following from Fetters et al., ${ }^{24}$ a change in packing length will yield the following effect on the original $G_{N 0}$ and disengagement time (i.e. reptation time), $\tau_{d 0}$,

$$
\begin{aligned}
G_{N} & =\frac{G_{N 0}}{\alpha^{3}} \\
\tau_{d} & =\frac{\tau_{d 0}}{\alpha^{6.8}} .
\end{aligned}
$$

Therefore, by knowing $f, \alpha$ can be calculated via Eq. 2 and the expected change in plateau modulus and disengagement time can be easily calculated via Eqs. 4 and 5 . Note that a small change in $\alpha$ has a very large effect on $G_{N}$ and an even larger effect on $\tau_{d}$.

The above argument assumes that the Kuhn length does not change with a change in side group chemistry. It is not clear that this is a good assumption and therefore we think it is best to demonstrate that this assumption is in fact supported by our current knowledge of polymer physics. Suppose that we have a backbone of poly(ethylene), PE, and could somehow put a benzene ring on every second C-atom, forming poly(styrene). Using Fetters et al. this would result in a change of Kuhn length from $13.7 \dot{A}$, see Table in Fetters, to 18 $\dot{A}$, calculated using equation:

$$
b=\frac{<R^{2}>_{0}}{M} \frac{m_{b}}{l \cos (\theta / 2)}
$$

where $<R^{2}>_{0} / M=0.437, m_{b}=54, \cos (\theta / 2)=0.83$ for CC bond angle, and $l=1.5 \dot{A}$ for CC bond length. ${ }^{24}$ This means that going from PE to PS only yields a $30 \%$ change in 
Kuhn length, this is in comparison to the $130 \%$ change in the packing length, $p$, estimated from Eq. 3. One could additionally argue that going from PE to PS is a dramatic change compared to the chemical change from PAA to PnBA, since the chemical change for PnBA is further away from the CC backbone. These arguments strongly support the assumption of a constant Kuhn length. Therefore, it can be argued that the major influence of changing the chemistry will be the ability to tie knots, i.e. the entanglement length or packing length.

If our simple arguments are correct, then the observed changes in LVE behavior (i) and (ii) above, should be accounted for by changes in chemistry and not hydrogen bonding between chains. The Baumgaertel-Schausberger-Winter (BSW) relaxation spectrum is a model that has been shown to accurately fit and predict LVE behavior of monodisperse entangled linear polymer melts. The BSW model has five parameters: $n_{e}$ and $n_{g}$, which represent empirical slopes of $G^{\prime \prime}$ immediately after $\omega_{L}$ and immediately before $\omega_{H}$, respectively, and $G_{N}, \tau_{d}$, and $\tau_{c}$, which are related to the theoretical tube model parameters: plateau modulus, disengagement time, and the relaxation time of one entanglement, respectively. ${ }^{25}$ For a monodisperse linear polymer, $n_{e}=-0.23$ and $n_{g}=0.7$. We perform a nonlinear least squares fit of the BSW model to the LVE data of PnBA in order to determine the values of $G_{N 0}, \tau_{d 0}$, and $\tau_{c}$. The best fit parameters are given in Table 2. The best fit BSW spectrum is plotted in Fig. 4a. It is evident from this figure that the BSW model fits the data very well, except in the terminal regime below $\omega_{L}$, as expected.

Using Eqs. 4 and 5, we can predict the BSW parameters as a function of AA fraction. The predicted values are given in Table 2. Fig. 4b-4d shows LVE data for samples AA6, AA13, and AA38 along with the BSW predictions accounting for the number of AA side groups per chain. The excellent agreement between the BSW predictions and the experimental data shows that our simple model is able to predict quantitatively the observed change in $G_{N}$ for increasing number of AA side groups. Furthermore, the simple model is able to capture the increase in the low frequency crossover as seen from Fig. 4a-4b. This is less evident in Fig. 4c-4d due to the convolution that occurs at low frequency for increased AA content. 
However, it is clear that that low frequency crossover is decreasing in the experimental data, which is the same trend predicted by Eq. 5, i.e. increasing $\tau_{d}$. One can conclude from these results that observations (i) and (ii) are strongly due to the geometric effects inherent in the hydrolysis of nBA to AA and not the resulting hydrogen bonding. However, changes in packing length cannot explain observation (iii) whereby a change in power law dependence of $G^{\prime}$ and $G^{\prime \prime}$ occurs. We therefore conclude that hydrogen bonding between chains is giving rise to this particular phenomenon. With regard to the data presented by Stadler and Freitas, we hypothesize that if one could account for how $G_{N 0}$ and $\tau_{d}$ would change with their chemistry, the same conclusions would be drawn.

Table 2: The parameters for PnBA were determined from nonlinear least squares fit. Parameters for AA6, AA13, and AA38 were calculated using Eqs. 4 and 5 using PnBA parameters taking into account that PnBA has 3\% AA. The theoretically pure PnBA can be calculated using the parameters for $3 \%$ AA to be $G_{N}=1.64 \times 10^{5} \mathrm{~Pa}$ and $\tau_{m}=0.97 \mathrm{~s}$, which is then used to make the parameter estimations for AA6, AA13, and AA38.

\begin{tabular}{l|c|c|c|c|c}
\hline \multicolumn{7}{c}{ BSW Parameters } \\
\hline & $\mathbf{G}_{N}[\mathrm{~Pa}]$ & $\tau_{d}[\mathrm{~s}]$ & $\tau_{c}[\mathrm{~s}]$ & $n_{e}$ & $n_{g}$ \\
\hline PnBA(3\% AA) & $1.71 \times 10^{5}$ & 1.06 & $3.5 \times 10^{-4}$ & -0.23 & 0.7 \\
AA6 & $1.78 \times 10^{5}$ & 1.16 & $3.5 \times 10^{-4}$ & -0.23 & 0.7 \\
AA13 & $1.92 \times 10^{5}$ & 1.45 & $3.5 \times 10^{-4}$ & -0.23 & 0.7 \\
AA38 & $2.84 \times 10^{5}$ & 3.37 & $3.5 \times 10^{-4}$ & -0.23 & 0.7 \\
\hline
\end{tabular}

Assuming that superposition holds, we subtract the corresponding BSW spectrum from the experimental data in order to examine the hydrogen bonding contribution to linear viscoelasticity. The remaining $G^{\prime}$ and $G^{\prime \prime}$ contribution due to hydrogen bonding is shown in Fig. 5a and 5b, respectively. Note that in these figures a larger frequency window for the data set is included than shown in Figure 1. The solid black line is an indicator of 0.5 power law scaling, which is indicative of a gel or Rouse dynamics. Such physics have been incorporated into a model known as the sticky Rouse model. ${ }^{26,27}$ In Fig. 5a, the power law scaling of $G^{\prime}$ for PnBA is slightly greater than 0.5 and decreases with increasing AA content. In Fig. 5b, the power law of $G^{\prime \prime}$ for PnBA is also slightly higher than 0.5 and decreases with increasing AA content. For sample AA38, the power law scaling of $G^{\prime}$ and $G^{\prime \prime}$ appears to be 


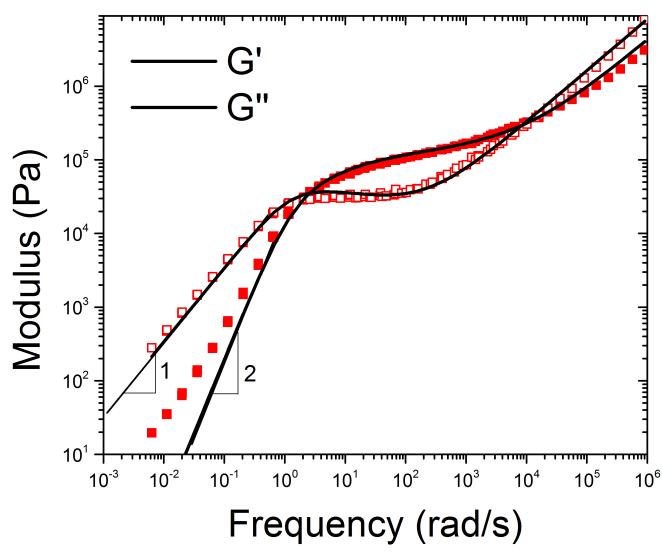

(a) $\mathrm{PnBA}$

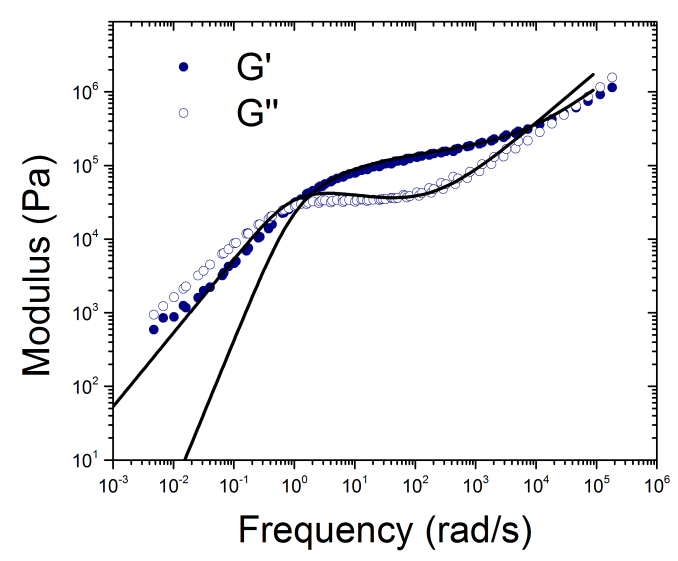

(c) AA13, $13 \% \mathrm{AA}$

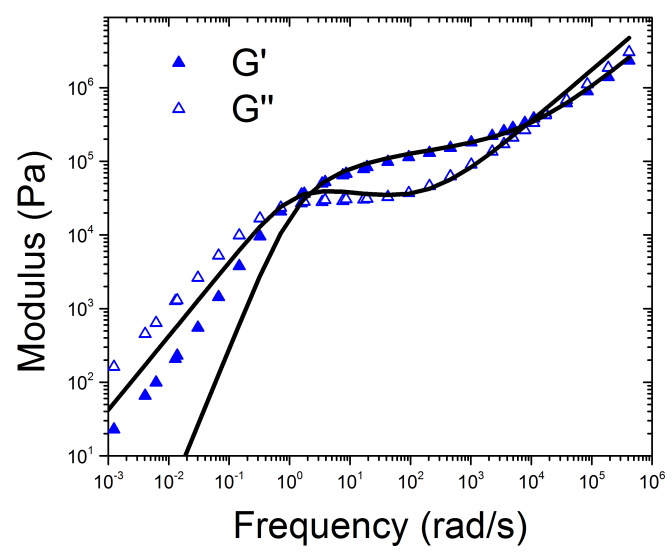

(b) AA6, 6\% AA

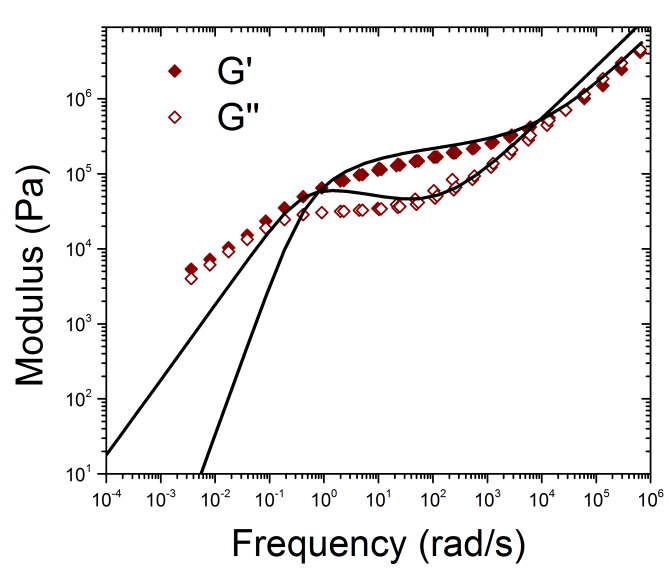

(d) AA38, 38\% AA

Figure 4: shows the linear viscoelastic response of all four samples measured using small amplitude oscillatory shear as presented in Fig. 1 along with BSW predictions (solid lines) assuming Eq. 4 and 5 hold.

precisely 0.5. Moreover, $G^{\prime}$ and $G^{\prime \prime}$ overlap each other for samples AA13 and AA38, which is indicative of a gel or Rouse dynamics.

Another observation from Fig. 5a and Fig. 5b is that there appears to be a steady increase in the magnitude of $G^{\prime}$ and $G^{\prime \prime}$ as a function of the number of AA groups per chain. Fig. 6a shows the magnitude of $G^{\prime}$ plotted as a function of the number of AA groups per chain for two frequencies. There appears to be a linear dependence of $\left|G^{\prime}\right|$ on the number of AA groups per chain for both frequencies. This linear dependence is in stark contrast to the highly nonlinear dependence of extensional stress as a function of the number of 
AA groups/chain in nonlinear rheology. Fig. 6b shows the extensional stress taken at the plateau seen in Fig. 3 plotted as a function of the number of AA groups for different strain rates. It is evident from this figure that this dependence is a strong function of strain rate. For example, for a $\dot{\epsilon}=0.01 \mathrm{~s}^{-1}$ the dependence appears linear for the concentrations of AA examined. However, for $\dot{\epsilon}=0.003 \mathrm{~s}^{-1}$ the trend is concave and for $\dot{\epsilon}=0.1 \mathrm{~s}^{-1}$ the trend is convex for increasing values of $f$.

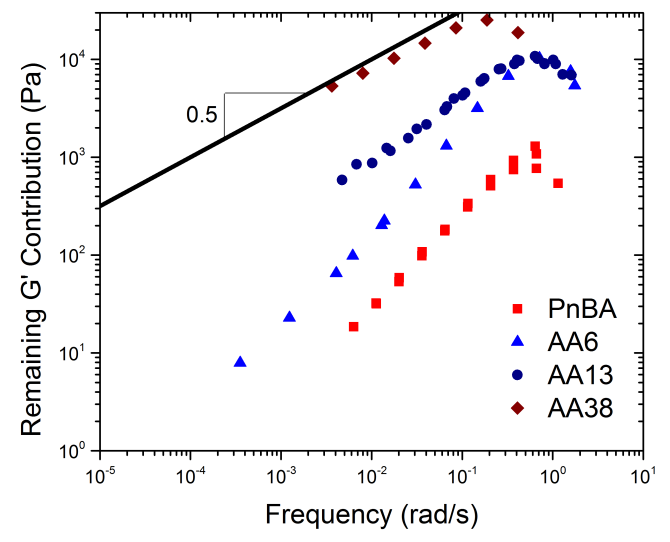

(a)

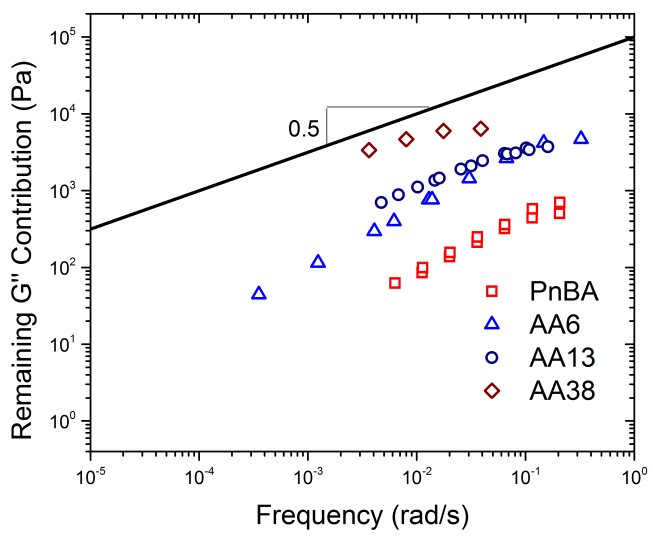

(b)

Figure 5: shows the remaining contribution of (a) $G^{\prime}$ and (b) $G^{\prime \prime}$ to LVE after subtraction of the corresponding BSW spectrum, see Fig. 4. The solid line indicates a power law scaling of 0.5 .

We speculated in the results section that the deviation of PnBA LVE from the classical power law scaling in the terminal regime was due to the presence of hydrogen bonding. It is clear from the results presented here that indeed hydrogen bonding for a monodisperse linear polymer manifests itself in the terminal regime of LVE. Surprisingly, hydrogen bonding has little effect on the LVE at frequencies above $\omega_{L}$. This suggests that the relaxation time of hydrogen bonding is slower than the inverse disengagement time of the polymer chains and therefore at frequencies larger than $1 / \tau_{d}$ the dynamics of the hydrogen bonds are irrelevant and classical polymer dynamics of the entangled chains dominate. This important observation is also evident in the nonlinear response of the samples. In Fig. 3, one observes that the largest strain hardening is observed for the slowest strain rates. In other words, 


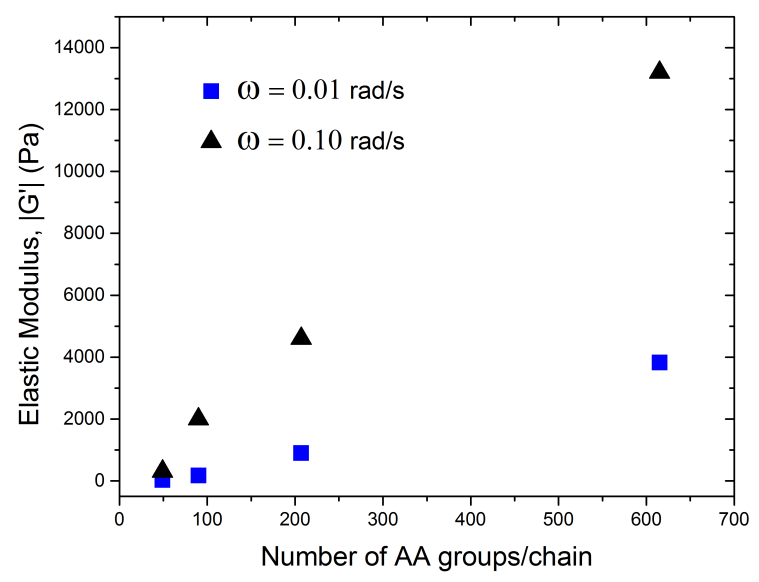

(a)

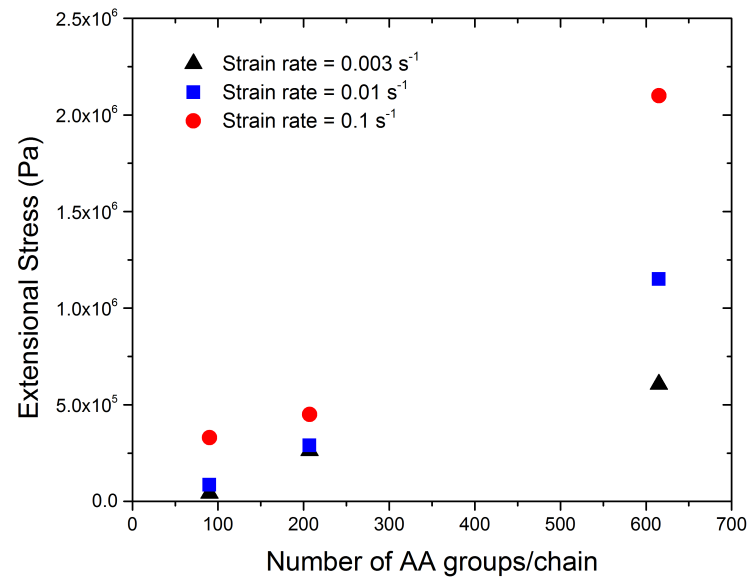

(b)

Figure 6: (a) shows the magnitude of $G^{\prime}$ as a function of the number of AA groups per chain for the given frequency shown in the legend and (b) shows the magnitude of extensional stress at the plateau as a function of the number of AA groups per chain for the given strain rate shown in the legend.

when the strain rate is much less than $1 / \tau_{d}$ the hydrogen bonds contribute strongest to the stress. However, for increasing strain rate, e.g. $\dot{\epsilon}=0.1 \mathrm{~s}^{-1}$, sample AA6, AA13, and AA38 show decreasing degrees of strain hardening. Note that these conclusions can only hold for the number of AA side groups studied here, i.e. up to $38 \%$.

Recall from the introduction that Lewis and coworkers in their study considering four different hydrogen bonding groups concluded that dimerization of a single side group was not enough to induce the formation of a network. This trend seems to hold true in the results presented here for the weakly entangled networks and low fraction of AA side groups studied by them. However, it is evident from this study that for an entangled linear polymer network with more than $12 \%$ AA side groups, the formation of a weak network/gel is possible. It is still not clear whether this is due to the large percentage of AA side groups or the fact that the chains are sufficiently entangled. The mechanism responsible for the network/gel formation is the subject of a future article, whereby the data is discussed in terms of a physical model. 


\section{Conclusions}

We present linear and nonlinear rheological results for a model monodisperse linear polymer, PnBA, which has been hydrolyzed to different extents to determine the impact of hydrogen bonding on polymer dynamics. There are two contributions to the observed trends in linear viscoelasticity as a function of increasing AA side groups, (i) changes in packing length and (ii) hydrogen bonding between chains. Using a simple model with no adjustable parameters, we are able to account for changes in packing length and therefore explicitly show the effect of hydrogen bonding. Hydrogen bonding specifically alters the power law dependence of $G^{\prime}$ and $G^{\prime \prime}$ in the LVE terminal regime, i.e. frequencies below $\omega_{L}$, of an entangled linear polymer network. More specifically, hydrogen bonding increases the magnitude of $G^{\prime}$ with respect to $G^{\prime \prime}$ such that at sufficiently high concentrations, i.e. $12 \%$ in our case, $G^{\prime}$ and $G^{\prime \prime}$ are parallel (power law 0.5) and equal in magnitude. For frequencies above $\omega_{L}$, hydrogen bonding has little to no effect on the LVE response. This suggests that there is a relaxation time associated with the hydrogen bonding groups that is greater than $1 / \omega_{L}$. For frequencies above $\omega_{L}$ the hydrogen bonds are too slow to effect LVE behavior. Extensional rheology was used to probe the contribution of hydrogen bonding to nonlinear dynamics. It was evident that for strain rates below $\omega_{L}$, the samples exhibited impressive strain hardening behavior. The magnitude of strain hardening decreases as the strain rate approached $\omega_{L}$. This trend supports the hypothesis that above a critical strain rate the hydrogen bonds become irrelevant. There is a need for better understanding of the action and strength of hydrogen bonding. Hopefully the data and analysis presented here will guide the foundation of theory work in this area.

\section{Acknowledgement}

The authors would like to thank Charles-Andre Fustin for guidance and suggestions regarding the determination of acrylic acid content after hydrolyzation. This work was funded by the People Programme (Marie Curie Actions) of the European Union's Seventh Framework 
Programme (FP7/2007-2013) under REA grant agreement no. 607937. E.vR. is a research associate of the FRS-FNRS. H.G. thanks FRIA for financial support.

\section{Supporting Information Available}

\section{References}

(1) Lewis, C.; Stewart, K.; Anthamatten, M. Macromolecules 2014, 47, 729-740.

(2) Lange, R. F. M.; Van Gurp, M.; Meijer, E. W. Journal of Polymer Science Part A: Polymer Chemistry 1999, 37, 3657-3670.

(3) Nair, K. P.; Breedveld, V.; Weck, M. Macromolecules 2008, 41, 3429-3438.

(4) Nair, K.; Breedveld, V.; Weck, M. Macromolecules 2011, 44, 3346-3357.

(5) Feldman, K. E.; Kade, M. J.; Meijer, E. W.; Hawker, C. J.; Kramer, E. J. Macromolecules 2009, 42, 9072-9081.

(6) Montarnal, D.; Tournilhac, F.; Hidalgo, M.; Couturier, J.-L.; Leibler, L. Journal of the American Chemical Society 2009, 131, 7966-7.

(7) Sivakova, S.; Bohnsack, D. a.; Mackay, M. E.; Suwanmala, P.; Rowan, S. J. Journal of the American Chemical Society 2005, 127, 18202-11.

(8) Rossow, T.; Seiffert, S. Polymer Chemistry 2014, 5, 3018.

(9) Mugemana, C.; Guillet, P.; Hoeppener, S.; Schubert, U. S.; Fustin, C.-A.; Gohy, J.-F. Chemical communications (Cambridge, England) 2010, 46, 1296-8.

(10) Eisenberg, A.; Kim, J. Introduction to Ionomers; Wiley, 1998; p 325.

(11) Chen, Q.; Tudryn, G.; Colby, R. Journal of Rheology (1978-present ... 2013, 16802, 1441-1462. 
(12) Zhang, L.; Brostowitz, N. R.; Cavicchi, K. a.; Weiss, R. a. Macromolecular Reaction Engineering 2014, 8, 81-99.

(13) Weiss, R. a.; Yu, W.-C. Macromolecules 2007, 40, 3640-3643.

(14) Sijbesma, R.; Meijer, E. Current opinion in colloid $\&$ interface science 1999, 4, 24-32.

(15) Brunsveld, L.; Folmer, B. J. B.; Meijer, E. W.; Sijbesma, R. P. Chemical Reviews 2001, 101, 4071-4098.

(16) Corbin, P.; Zimmerman, S. Journal of the American Chemical Society 1998, 7863, 9710-9711.

(17) Yan, X.; Wang, F.; Zheng, B.; Huang, F. Chemical Society reviews 2012, 41, 6042-65.

(18) Stadler, R.; de Lucca Freitas, L. Colloid and Polymer Science 1988, 266, 1102-1109.

(19) Stadler, R.; Freitas, L. D. L. Polymer Bulletin 1986, 15, 173-179.

(20) Leong, K.; Butler, G. Journal of Macromolecular Science A. 1980, 14, 287.

(21) Jullian, N.; Leonardi, F.; Grassl, B.; Peyrelasse, J.; Derail, C. Appl. Rheol 2010, 20, $1-11$.

(22) Román Marín, J. M.; Huusom, J. K. b.; Alvarez, N. J.; Huang, Q.; Rasmussen, H. K.; Bach, A.; Skov, A. L.; Hassager, O. Journal of Non-Newtonian Fluid Mechanics 2013, $194,14-22$.

(23) Sridhar, T.; Acharya, M.; Nguyen, D. A.; Bhattacharjee, P. K. Macromolecules 2014, $47,379-386$.

(24) Fetters, L.; Lohse, D.; Colby, R. Physical properties of polymers . . 2007,

(25) Huang, Q.; Mednova, O.; Rasmussen, H. K.; Alvarez, N. J.; Skov, A. L.; Almdal, K.; Hassager, O. Macromolecules 2013, 46, 5026-5035. 
(26) Rubinstein, M.; Semenov, A. Macromolecules 2001, 34, 1058-1068.

(27) Rubinstein, M.; Semenov, A. Macromolecules 1998, 31, 1386-1397.

This material is available free of charge via the Internet at http://pubs.acs.org/. 